\title{
Tendencia en la incidencia del cáncer testicular de células germinales en un área sanitaria española de 300.000 habitantes (1991-2005)
}

\author{
Llanes González L, Luján Galán M, Rodríguez García N, García Tello A, Berenguer Sánchez A. \\ Servicio de Urología. Hospital Universitario de Getafe. Madrid.
}

Actas Urol Esp. 2008;32(7):691-695

\section{RESUMEN}

TENDENCIA EN LA INCIDENCIA DEL CÁNCER TESTICULAR DE CÉLULAS GERMINALES EN UN ÁREA SANITARIA ESPAÑOLA DE 300.000 HABITANTES (1991-2005)

Objetivo: Describir las características y tendencia temporal de los cánceres testiculares en un área sanitaria española.

Material y métodos: Se realizó una búsqueda de todos los pacientes residentes en nuestra área sanitaria diagnosticados de cáncer testicular por primera vez. Se calcularon los datos de incidencia, cambio porcentual anual (APC) según tasa de incidencia ajustada a la población española, edad al diagnóstico y las frecuencias de los diferentes tipos histológicos tumorales.

Resultados: La incidencia del cáncer testicular pasó de 0,84/100.000 varones en 1991 a 1,91/100.000 varones en 2005, con un pico de 6,77/100.000 varones en 2003. El cambio porcentual anual (APC) ajustado a la población española se calculó en 2,39\%. Por subtipos histológicos, para los seminomas: la tasa de incidencia pasó de 0,84 a 0,64/100.000 (1991-2005), con un pico de 5,41/100,000 en 2003 y un APC ajustado a la población española de 6,06. Para los no seminomas, la tasa de incidencia varió de 1,66 a 1,28/100.000 (1992-2005), con un pico de 3,65/100.000 en 2001 y un APC ajustado a la población española de 12,74. La edad al diagnóstico fue, para los seminomas: media 31,23, DE 8,56, mediana 30; y para los tumores no seminomatosos: media 23,68, DE 6,85, mediana 25. La comparación de las medias de ambas edades resultó estadísticamente significativa (p، 0,0001).

Discusión: Existe un aumento de la incidencia del cáncer testicular de células germinales en nuestro medio, tal y como se ha demostrado en otros países.

Palabras clave: Neoplasias testiculares. Incidencia. Epidemiología.

\section{ABSTRACT \\ TRENDS IN THE INCIDENCE OF TESTICULAR GERM CELL CANCER IN A 300.000 INHABITANTS SPANISH POPULATION (1991-2005)}

Objective: To describe the features and the time trends of the testicular cancer in a spanish population.

Materials and methods: Data on incident cases of testicular germ cell cancer diagnosed in our population were extracted from the Cancer Registry of our Department. We calculed annual incidence rates of testicular cancer, the Spanish population-adjusted annual percent change (APC), age of diagnosis and the different histologic types frequencies.

Results: The overall incidence rate rose from 0.84 per 100,000 males to 1.91 per 100,000 males from 1991 till 2005 , with a peak of 6.77 per 100,000 males in 2003. The Spanish population-adjusted APC was 2.39\%. By histologic subgroup, for seminomas: the incidence rate varied from 0.84 per 100,000 males to 0.64 per 100,000 (1991-2005), with a peak of 5.41 per 100,000 in 2003 and the Spanish population-adjusted APC was 6.06. For nonseminomas, the incidence rate varied from 1.66 per 100,000 to 1.28 per 100,000 (1992-2005), with a peak of 3.65 per 100.000 in 2001 and the Spanish population-adjusted APC was 12.74. Mean ages were 31.23 years (median 30, SD 8.56) for seminomas, and 23.68 years (median 25, SD 6.85) for nonseminomas, with statistic significance (p 0.0001 ).

Discussion: The increasing testicular cancer incidence observed for this population follows the time trends showed in other European countries.

Keywords: Testicular neoplasms. Incidence rate. Epidemiology. 
$\mathrm{E}^{1}$ cáncer de testículo de células germinales en sus diferentes variedades histológicas es una neoplasia del varón joven. En los países del norte de Europa, Reino Unido, Canadá, Nueva Zelanda, Australia y los Estados Unidos se ha demostrado una tendencia al aumento de la incidencia de este tumor a lo largo del siglo XX. Los diferentes estudios realizados no han podido aportar luz sobre los posibles factores de riesgo ni sobre sus causas, por lo que no existe en la actualidad ninguna hipótesis que explique satisfactoriamente las tendencias en cuanto a la incidencia ni distribución geográfica de este cáncer. El ánimo de este trabajo es establecer la incidencia del cáncer testicular de células germinales y su tendencia temporal en un área sanitaria de la Comunidad de Madrid.

\section{MATERIAL Y MÉTODOS}

Para la obtención de los datos demográficos, se realizo una búsqueda de todos los pacientes residentes en nuestra área sanitaria diagnosticados de cáncer testicular por primera vez entre 1 de enero de 1992 y 31 de diciembre de 2004. Para ello, acudimos a la base de datos de hospitalización de nuestro servicio e identificamos a los individuos en base a su código de diagnostico o bien de procedimiento (código 186 de diagnostico, neoplasia maligna del testículo o código de procedimiento 62.3 , orquiectomía unilateral, de la Clasificación Internacional de Enfermedades, C.I.E. 9 ${ }^{a}$ revisión). Desechamos de la búsqueda inicial aquellos enfermos cuyo diagnostico anatomopatológico era diferente de un cáncer de células germinales, esto es tumores no germinales, tumores paratesticulares, torsiones testiculares, tuberculosis y otros procesos inflamatorios o de cualquier otra naturaleza, salvo la indicada.

A continuación, se calcularon los datos de incidencia, cambio porcentual anual (APC) según tasa de incidencia ajustada a la población española, edad al diagnóstico y las frecuencias de los diferentes tipos histológicos tumorales. Las siguientes definiciones aclaran los objetivos de nuestro trabajo:

- Tasa de incidencia de cáncer: numero de nuevos cánceres (de tipo o de localización específica) ocurridos en una población específica durante un año. Se expresa como numero de cánceres por 100.000 habitantes en riesgo.

- Cambio porcentual anual (annual percent change, APC): Se usa para medir la tendencia o el cambio en la tasa durante el tiempo. Es muy empleado para medir tendencias en tasas de enfermedad y mortalidad y se calcula usando un modelo lineal sobre las tasas ajustadas por edad ${ }^{1}$.

Las relaciones entre las características de las dos estirpes histológicas (seminomas y no seminomas) se llevaron a cabo mediante las pruebas de la $\mathrm{T}$ de Student, para la comparación de las medias de las variables cuantitativas y $\chi^{2}$ de Pearson para la comparación de proporciones. La recogida de datos y el análisis estadístico se llevaron a cabo con el programa SPSS 12.0. Todos los análisis utilizaron un nivel de significación de $\mathrm{p}<0,05$.

\section{RESULTADOS}

La Tabla 1 muestra la población existente en los municipios que conforman el área 10 sanitaria de la Comunidad de Madrid desde 1991 a 2005, según los datos del Instituto Nacional de Estadística $(\mathrm{INE})^{2}$. A lo largo del período de estudio, los habitantes se han incrementado en más de 74.000 personas, lo que supone un incremento aproximado del $31 \%$.

Las Tablas 2, 3 y 4 muestran las tasas de incidencia bruta y ajustadas a la población de la comunidad de Madrid, española y mundial por 100.000 varones, de cada tipo histológico tumoral.

Tabla 1. Población del área sanitaria 10 de la Comunidad Autónoma de Madrid (1991-2005)

\begin{tabular}{llll}
\hline & Varones & Mujeres & Total \\
\hline 1991 & 118.894 & 118.229 & 237.123 \\
1992 & 119.450 & 119.528 & 238.978 \\
1993 & 120.588 & 120.505 & 241.093 \\
1994 & 123.171 & 122.910 & 246.081 \\
1995 & 125.578 & 125.263 & 250.841 \\
1996 & 126.694 & 126.375 & 253.069 \\
1997 & 124.070 & 124.975 & 249.045 \\
1998 & 126.806 & 127.407 & 254.213 \\
1999 & 129.291 & 129.869 & 259.160 \\
2000 & 131.786 & 132.244 & 264.030 \\
2001 & 137.015 & 136.864 & 273.879 \\
2002 & 142.903 & 141.810 & 284.713 \\
2003 & 147.813 & 146.423 & 294.236 \\
2004 & 151.395 & 150.119 & 301.514 \\
2005 & 156.820 & 154.648 & 311.468 \\
\hline
\end{tabular}


Tabla 2. Tasas de incidencia bruta y ajustadas a la población de la comunidad de Madrid, española y mundial por 100.000 varones, para el global de cánceres testiculares.

\begin{tabular}{|c|c|c|c|c|}
\hline \multicolumn{5}{|c|}{$\begin{array}{l}\text { Resumen incidencia cáncer testicular área } 10 \text { de la } \\
\text { CAM }\end{array}$} \\
\hline & Bruta & $\begin{array}{l}\text { Ajustada } \\
\text { CAM }\end{array}$ & $\begin{array}{l}\text { Ajustada } \\
\text { España }\end{array}$ & $\begin{array}{l}\text { Ajustada } \\
\text { Mundo }\end{array}$ \\
\hline 1991 & 0,84 & 0,92 & 0,92 & 0,75 \\
\hline 1992 & 2,49 & 2,27 & 2,25 & 2,16 \\
\hline 1993 & 1,62 & 1,47 & 1,51 & 2,21 \\
\hline 1994 & 0,80 & 0,85 & 0,82 & 0,71 \\
\hline 1995 & 0,79 & 0,65 & 0,64 & 0,68 \\
\hline 1996 & 1,58 & 1,37 & 1,32 & 1,32 \\
\hline 1997 & 3,22 & 2,85 & 2,85 & 3,88 \\
\hline 1998 & 2,37 & 2,15 & 2,09 & 1,96 \\
\hline 1999 & 0,00 & 0,00 & 0,00 & 0,00 \\
\hline 2000 & 3,04 & 2,56 & 2,36 & 2,26 \\
\hline 2001 & 5,84 & 5,18 & 5,01 & 5,15 \\
\hline 2002 & 1,40 & 1,15 & 1,09 & 1,11 \\
\hline 2003 & 6,77 & 6,04 & 5,63 & 6,05 \\
\hline 2004 & 4,62 & 3,98 & 3,85 & 4,15 \\
\hline 2005 & 1,91 & 1,94 & 1,86 & 1,66 \\
\hline
\end{tabular}

Tabla 3. Tasas de incidencia bruta y ajustadas a la población de la comunidad de Madrid, española y mundial por 100.000 varones, para seminomas.

\begin{tabular}{lcccc}
\hline \multicolumn{6}{l}{ Resumen incidencia seminoma área 10 de la CAM } \\
\hline & Bruta & $\begin{array}{c}\text { Ajustada } \\
\text { CAM }\end{array}$ & $\begin{array}{c}\text { Ajustada } \\
\text { España }\end{array}$ & $\begin{array}{c}\text { Ajustada } \\
\text { Mundo }\end{array}$ \\
\hline 1991 & 0,84 & 0,92 & 0,92 & 0,75 \\
1992 & 0,83 & 0,91 & 0,91 & 0,74 \\
1993 & 0,00 & 0,00 & 0,00 & 0,00 \\
1994 & 0,80 & 0,85 & 0,82 & 0,71 \\
1995 & 0,00 & 0,00 & 0,00 & 0,00 \\
1996 & 0,79 & 0,65 & 0,64 & 0,68 \\
1997 & 0,81 & 0,73 & 0,75 & 1,10 \\
1998 & 0,79 & 0,78 & 0,78 & 0,65 \\
1999 & 0,00 & 0,00 & 0,00 & 0,00 \\
2000 & 1,52 & 1,50 & 1,43 & 1,29 \\
2001 & 2,19 & 6,25 & 7,05 & 2,90 \\
2002 & 0,00 & 0,00 & 0,00 & 0,00 \\
2003 & 5,41 & 4,80 & 4,48 & 4,21 \\
2004 & 1,98 & 1,69 & 1,63 & 1,67 \\
2005 & 0,64 & 0,68 & 0,66 & 0,57 \\
\hline & & & &
\end{tabular}

Tabla 4. Tasas de incidencia bruta y ajustadas a la población de la comunidad de Madrid, española y mundial por 100.000 varones, para no seminomas.

\begin{tabular}{|c|c|c|c|c|}
\hline \multicolumn{5}{|c|}{ Resumen incidencia no seminoma área $10 \mathrm{CAM}$} \\
\hline & Bruta & $\begin{array}{l}\text { Ajustada } \\
\text { CAM }\end{array}$ & $\begin{array}{c}\text { Ajustada } \\
\text { España }\end{array}$ & $\begin{array}{c}\text { Ajustada } \\
\text { Mundo }\end{array}$ \\
\hline 1991 & 0,00 & 0,00 & 0,00 & 0,00 \\
\hline 1992 & 1,66 & 1,36 & 1,34 & 1,42 \\
\hline 1993 & 1,62 & 1,47 & 1,51 & 2,21 \\
\hline 1994 & 0,00 & 0,00 & 0,00 & 0,00 \\
\hline 1995 & 0,79 & 0,65 & 0,64 & 0,68 \\
\hline 1996 & 0,79 & 0,72 & 0,68 & 0,64 \\
\hline 1997 & 2,42 & 2,12 & 2,10 & 2,78 \\
\hline 1998 & 1,58 & 1,37 & 1,31 & 1,32 \\
\hline 1999 & 0,00 & 0,00 & 0,00 & 0,00 \\
\hline 2000 & 1,52 & 1,24 & 1,14 & 1,10 \\
\hline 2001 & 3,65 & 3,20 & 3,07 & 3,33 \\
\hline 2002 & 1,40 & 1,15 & 1,09 & 1,11 \\
\hline 2003 & 1,35 & 1,24 & 1,15 & 1,84 \\
\hline 2004 & 2,64 & 2,29 & 2,21 & 2,48 \\
\hline 2005 & 1,28 & 1,26 & 1,20 & 1,09 \\
\hline
\end{tabular}

Las Figuras 1,2 y 3 representan la variación a lo largo del período de estudio de las diferentes tasas de incidencia del cáncer de testículo (global y por subtipos tumorales).

El cambio porcentual anual (APC) según la tasa de incidencia ajustada a la población española se calculó en un 2,39\%, con un rango de -9,04 a 15,26 $(\mathrm{p}<0,05)$, para el global de cánceres de testículo. Si desglosamos por tipo tumoral, los seminomas tuvieron un APC de 6,06; rango de $-12,37$ a 28,37 $(\mathrm{p}<0,05)$ y los tumores no seminomatosos: $\mathrm{APC}=12,74$; rango de $-3,85$ a $32,20(\mathrm{p}<0,05)$.

En cuanto a la edad al diagnóstico, para los seminomas fue de media 31,23 años, desviación estádar (DE) 8,56, mediana 30 y rango (19-50). Para los tumores no seminomatosos, la edad media fue de 23,68 años, $\mathrm{DE}=6,85$, mediana 25 y rango (135). La comparación de las medias de ambas edades (T de Student) resultó estadísticamente significativa (p، 0,0001).

La histología tumoral se dividió en: seminomas $47,9 \%(23 / 48)$ y no seminomas $52,1 \%(25 / 48)$. A su vez los tumores no seminomatosos fueron puros: $28 \%(7 / 25)$, mixtos: $72 \%(18 / 25)$ y tumores fundidos: $8 \%(2 / 25)$. La lateralidad de las neoplasias fue 


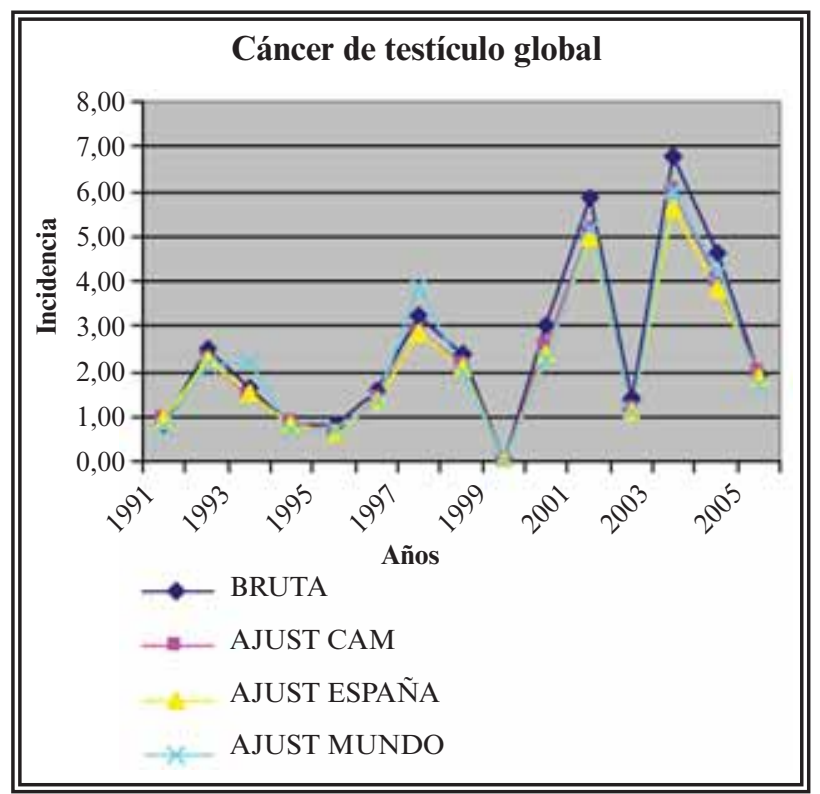

FIGURA 1. Variación a lo largo del periodo de estudio de las diferentes tasas de incidencia del cáncer de testiculo global.

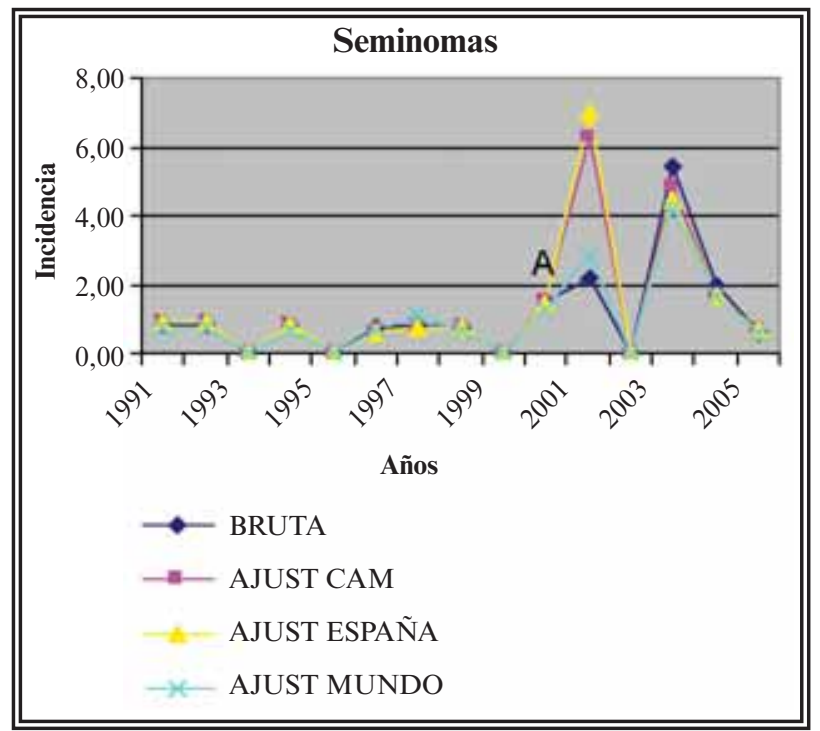

FIGURA 2. Variación a lo largo del periodo de estudio de las diferentes tasas de incidencia del cáncer de testiculo (Seminoma).

en los seminomas: $75 \%$ derecho y $25 \%$ izquierdo y en los tumores no seminomatoso: $66,6 \%$ derecho y $33,3 \%$ izquierdo. La comparación de estas proporciones ( $\chi^{2}$ de Pearson) no demostró diferencias en cuanto al lado de aparición del tumor $(\mathrm{p}=0,65)$.

\section{DISCUSIÓN}

Aunque no existen registros universales de cáncer, se puede estimar la tasa de incidencia en base

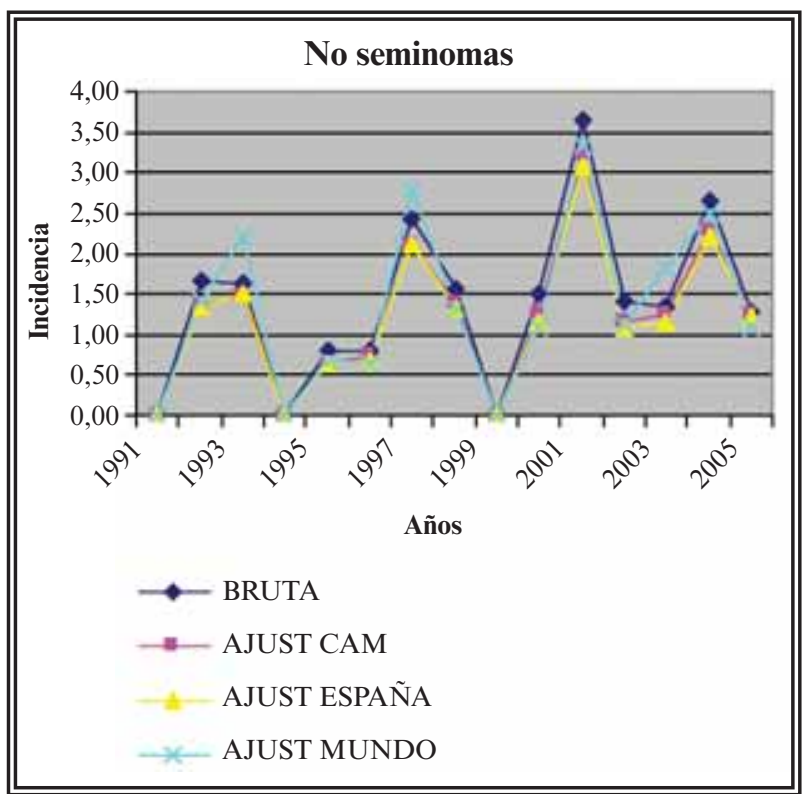

FIGURA 3. Variación a lo largo del periodo de estudio de las diferentes tasas de incidencia del cáncer de testículo (No Seminoma).

a los registros nacionales, regionales o locales de incidencia y de mortalidad. En nuestro estudio hemos utilizado un registro local, el de los diagnósticos de cáncer de testículo, acaecidos en el Hospital Universitario de Getafe que proporciona la atención especializada del área 10 sanitaria de la Comunidad de Madrid. Este trabajo representa un buen escenario para establecer las tasas de incidencia en el área de Madrid porque nuestro hospital pertenece al Sistema Nacional de Salud (SNS), siendo el único centro existente en un área de aproximadamente 300.000 habitantes, por lo que la mayor parte de los diagnósticos son realizados en nuestro departamento. Además, y teniendo en cuenta: el perfil socioeconómico de la población (en su mayoría trabajadores por cuenta ajena y de clase media-baja), las características del SNS (universal y gratuito), nos hace sospechar que el número de consultas privadas fuera del ámbito hospitalario sean muy bajas. Por ello consideramos que las tasas de incidencia calculadas son una estimación aceptable, aunque los resultados deben tomarse con precaución.

Nuestros hallazgos fundamentales son dos: $\mathrm{El}$ primero, que la incidencia del cáncer de testículo se está incrementando. Y el segundo, que esta tendencia temporal es similar para ambos tipos tumorales, más marcado en los tumores no seminomatosos que en los seminomas y que por tanto, la(s) causa(s) 
desconocida(s) responsable(s) de este incremento es compartida por las dos formas histológicas. Diferentes estudios han demostrado que probablemente esa(s) causa(s) haya que buscarla(s) en eventos tempranos en la vida de los individuos afectos, puesto que 1) el pico de incidencia del cáncer de testículo se da en varones jóvenes, 2) el cáncer in situ, precursor de todos los tumores germinales, se cree que se genera durante el desarrollo intrauterino; 3) el riesgo de cáncer testicular se asocia con la cohorte de nacimiento; y 4) los estudios de población inmigrante demuestran que los hombres mantienen la tasa de incidencia de su lugar de origen, independientemente de la edad de inmigración ${ }^{3,4}$. Además, parece fuera de toda duda el papel crítico de los andrógenos, puesto que aparte del pequeño pico infantil, los tumores testiculares desaparecen en la época pre-puberal y se incrementan rápidamente después de la pubertad ${ }^{5}$.

Estos dos hallazgos ya han sido comunicados recientemente en estudios de incidencia en países del norte de Europa, en los cuales se ha demostrado que esta tendencia incremental de la incidencia del cáncer de testículo es un fenómeno de cohorte de nacimiento ${ }^{6}$, esto es, cuanto más aumenta la población más aumenta la incidencia del tumor, salvo en la cohorte de nacidos durante la Segunda Guerra Mundial en los países del Norte de Europa por razones evidentes.

La tendencia anual al aumento de la incidencia del cáncer de testículo se ha estimado en los diferentes trabajos en un 2,3\% en Suecia, 4,8\% en Polonia y $5,2 \%$ en la Antigua Alemania Oriental para el período 1945-19895. En Dinamarca del 2,6\% anual (19431996) ${ }^{5}$ y en Ontario (Canadá) del 2\% desde 1964 a $1996^{7}$. Desafortunadamente, no existen registros reales de la incidencia del cáncer testicular de células germinales en España. Recientes trabajos parecen demostrar que no existe una tendencia marcada al incremento en su incidencia ${ }^{9,10}$ y las cifras que ofrece la IARC (Internacional Agency for Research on Cancer), colocan a nuestro país en entre los países europeos de menor incidencia $(0,5 \text { a } 1,9 / 100.000)^{11}$. No obstante, las tasas de incidencia media del período estudiado en nuestra área sanitaria, tanto bruta $(2,53 / 100.000$ varones) como ajustada a la población española $(2,15 / 100.000$ varones) son menos optimistas y la tasa de crecimento anual nos acerca a ciertos países nórdicos como Suecia ${ }^{5}$.

\section{CONCLUSIONES}

Existe una tendencia al aumento de la incidencia del cáncer testicular de células germinales en nuestro medio, más marcado en los tumores no seminomatosos, tal y como se ha demostrado en otros países. Los seminomas aparecen a edades significativamente más tardías que los no seminomatosos, a pesar de que ambas son neoplasias del varón joven y además no hemos encontrado una tendencia a la lateralidad en la afectación neoplásica en ambos grupos tumorales.

\section{REFERENCIAS}

1. Fay MP, Tiwari RC, Feuer EJ, Zou Z. Estimating average annual percent change for disease rates without assuming constant change. Biometrics. 2006;62(3):847-854.

2. Instituto Nacional de Estadística. http://www.ine.es/. Último acceso 21/09/2007.

3. Richiardi L, Bellocco R, Adami HO, Torrång A, Barlow L, Hakulinen T, et al. Testicular cancer incidence in eight northern European countries: secular and recent trends. Cancer Epidemiol Biomarkers Prev. 2004;13(12):2157-2166.

4. Bray F, Richiardi L, Ekbom A., Forman D, Pukkala E, Cuninkova M, et al. Do testicular seminoma and noseminoma share the same etiology? Evidence from and age-period-cohort analysis of incidence trends in eight European countries. Cancer Epidemiol Biomarkers Prev. 2006;15(4):652-658.

5. Moller H. Trends in incidence of testicular cancer and prostate cancer in Denmark. Human reproduction. 2004;16(5):10071011.

6. Bergström R, Adami HO, Möhner M, Zatonski W, Storm H, Ekbom A, et al. Increase in testicular cancer incidence in six European countries: a birth cohort phenomenon. J Natl Cancer Inst. 1996;88(11):727-733.

7. Weir HK, Marrett LD, Moravan V. Trends in the incidence of testicular germ cell cancer in Ontario by histologic subgroup, 1964-1996. CMAJ. 1999;160(2):201-205.

8. Santos D, Páez A, Luján M, Llanes L, Escalera C, Pascual C, Berenguer A. Incidencia del cáncer urológico en un área sanitaria de 300.000 habitantes. Actas Urol Esp. 2004;28(9):646649.

9. Tabernero J, Paz-Ares L, Salazar R, Lianes P, Guerra J, Borrás $\mathrm{J}$, et al. Incidente of contralateral germ cell testicular tumors in South Europe: Report of the experience at 2 spanish university hospitals and review of the literature. J Urol. 2004;171(1):164167.

10. Huyghe E, Plante P, Thonneau PF. Testicular cancer variations in time and space in Europe. Eur Urol. 2007;51(3):621-628.

11. IARC. Cancer incidente in five continents; vol. 8. Lyon: IARC Scientific Publication; 2002.

Correspondencia autor: Dr. L. Llanes González

Sarasate, 27 - 28222 Majadahonda. Madrid.

E-mail autor: luis.llanes@yahoo.es

Información artículo: Original - Cáncer testículo

Trabajo recibido: noviembre 2007

Trabajo aceptado: abril 2008 